\title{
Enfermedad fúngica invasora (EFI) por hongos filamentosos en la Región de Valparaíso, Chile, desde la implementación del diagnóstico rápido de laboratorio
}

\author{
Rodrigo Cruz, Patricio Álvarez, Felipe Provoste, Karen Ducasse, \\ Marcela González, Gonzalo Wilson y Javier Díaz
}

\section{Invasive fungal disease (IFD) by filamentous fungi in the Valparaíso Region, Chile, since implementation of rapid laboratory diagnosis}

Introduction: Invasive fungal diseases (IFD) by filamentous fungi are a common cause of morbidity and mortality in immunocompromised patients, especially those with myeloid leukemia. In 2011 a protocol for the rapid diagnosis of IFD by filamentous fungi was implemented in Valparaiso Region. Objectives: To describe cases of IFD by filamentous fungi of the Valparaíso Region, since the implementation of rapid diagnosis and to compare results with the period 2004-2009. Materials and Method: Descriptive and prospective study conducted in two public hospitals: Carlos van Buren at Valparaiso and Gustavo Fricke at Viña del Mar. We selected patients with a diagnosis of filamentous fungal diseases considering the EORTC/MSG criteria. Demographics, underlying diseases, risk factors for EFI, galactomannan (GM) results in blood and bronchoalveolar lavage, cultures and biopsies, treatment and overall lethality rates at 30 days were registered. Results: Eighteen patients were detected, 6 with proven and 12 probable IFD. Nine were diagnosed by GM, 8 by culture and two with both methods. In cases which the agent (9/18) was isolated from Rhizopus oryzae was the most frequent. When comparing overall lethality with the period 2004-2009, there was a reduction of $47.8 \%$, which was statistically significant. Conclusions: Compared to data previously published in the region, demographic and comorbidities of patients with IFD caused by filamentous fungi are similar, however the currently rapid diagnosis protocol has improved survival of patients and lethality experienced overall decrease.

Key words: Invasive fungal disease, rapid diagnosis, lethality.

Palabras clave: Enfermedad fúngica invasora, diagnóstico rápido, letalidad.

\section{Introducción}

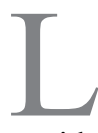

a enfermedad fúngica invasora (EFI) por hongos filamentosos es una causa frecuente de morbilidad y mortalidad en pacientes inmunocomprometidos $^{1,2}$. Entre los pacientes con mayor riesgo, tanto en población adulta como pediátrica, se encuentran aquellos que cursan con neutropenia secundaria a quimioterapia por leucemia, receptores de trasplantes de progenitores hematopoyéticos o de órganos sólidos, enfermedad injerto contra hospedero y usuarios de altas dosis y prolongadas de corticosteroides $\mathrm{u}$ otros inmunosupresores ${ }^{2,3}$.

Son varios los géneros y especies de hongos filamentosos que pueden provocar estas infecciones; el género Aspergillus y en especial A. fumigatus es el más frecuente en la mayoría de los trabajos publicados ${ }^{3,4}$. En Chile, en varias series de casos se ha demostrado que $A$ fumigatus es también la especie más aislada; sin embargo, otras como A. flavus, A. niger y A. nidulans han aumentado su frecuencia ${ }^{3,5,6}$. Géneros como Fusarium, Sarocladium, hongos dematiáceos y Mucorales se identifican con menor frecuencia ${ }^{7,8}$.

Los síntomas de la EFI son inespecíficos y la radiología convencional es de poca utilidad por la aparición tardía de las alteraciones, razón por la cual la tomografia axial computada (TAC) es el examen de elección por su alto valor predictor positivo 9 .

La detección de galactomanano (GM), componente de la pared celular de Aspergillus, se puede realizar mediante un ELISA con anticuerpos monoclonales EBA-2 de rata (Platelia Aspergillus ${ }^{\circledR}$, Bio-Rad, Francia) en el suero o en lavado broncoalveolar (LBA) de pacientes oncohematológicos, tanto en adultos como en niños ${ }^{10,11}$ y ha sido incluido como criterio diagnóstico de aspergilosis invasora probable en las definiciones de consenso de la EORTC/MSG ${ }^{11}$. Varios trabajos han mostrado que la sensibilidad de este examen es de aproximadamente $80 \%$ y la especificidad de $90 \%$, pudiendo ser detectada la enfermedad antes que las manifestaciones clínicas y radiológicas aparezcan, ayudando así al inicio precoz del tratamiento antifúngico ${ }^{10,12}$.
Universidad de Valparaíso, Valparaíso, Chile.

Cátedra de Micología (RC). Alumno de Medicina (PA, FP). Hospital Carlos van Buren de Valparaíso, Chile. Unidad de Infectología (RC, GW) Hospital Dr. Gustavo Fricke de Viña del Mar, Chile.

Unidad de Infectología (KD, MG) Unidad de Hematología (JD)

Los autores declaran no tener conflictos de interés.

Fuente de financiamiento: fondos de la Cátedra de Micología de la Universidad de Valparaíso.

Recibido: 23 de junio de 2014 Aceptado: 9 de diciembre de 2014

Correspondencia a:

Rodrigo Cruz Choappa rcruzchoappa@gmail.com 
El examen microscópico directo (EMD) y cultivo en agar Sabouraud tiene una sensibilidad entre 11 y $80 \%$, dependiendo del tipo de muestra, enfermedad de base y momento del diagnóstico de la infección; sin embargo, no siempre es posible tomar estas muestras en los pacientes $^{5,13}$.

En diciembre del 2011 se implementó en la Universidad de Valparaíso un protocolo para el diagnóstico rápido de la EFI por hongos filamentosos; el cual incluye detección de GM en sangre y en LBA, informe del EMD el mismo día de la obtención de la muestra, informe de las biopsias (BP) en 24 a 48 h de ser obtenidas y revisión diaria de los cultivos.

El objetivo del presente trabajo fue describir los casos de EFI por hongos filamentosos documentados desde la implementación de este protocolo para su diagnóstico rápido en la Región de Valparaíso y comparar la experiencia clínica con un período previo a su inicio: años 2004-2009.

\section{Materiales y Métodlo}

Estudio descriptivo y prospectivo. Ingresaron todos aquellos pacientes, adultos y niños, en quienes se diagnosticó un episodio de EFI por un hongo filamentoso durante el período comprendido entre diciembre de 2011 y marzo del 2014, en los hospitales públicos Carlos van Buren de Valparaíso y Dr. Gustavo Fricke de Viña del Mar. Los datos fueron obtenidos de las fichas clínicas de los pacientes e información aportada por los médicos tratantes. El diagnóstico fue realizado por el equipo tratante en cada caso particular, considerando los criterios EORTC/MSG ${ }^{11}$.

\section{Recopilación y análisis de datos}

En los casos identificados se completó una ficha que incluyó antecedentes demográficos, enfermedad de base, factores de riesgo conocidos para EFI por hongos filamentosos, imágenes de la TAC, número y resultados de GM en sangre, EMD, cultivos con desarrollo de hongos filamentosos y biopsia con presencia de hifas septadas o cenocíticas, tratamiento antifúngico y letalidad global a 30 días. La identificación de las especies aisladas se realizó por morfología y pruebas fisiológicas en la Cátedra de Micología de la Universidad de Valparaíso.

En el análisis estadístico se usó los programas Excel y Stata versión 11.1. Para determinar significancia estadística entre la letalidad global actual y la del período previo se usó prueba exacta de Fisher. Los resultados de las variables nominales se expresaron en porcentajes y de las numéricas en promedios.

\section{Resulltados}

Durante el período estudiado se identificaron 18 pacientes con diagnóstico de EFI por hongos filamentosos, de los cuales 11 fueron hombres mayores de 15 años, con una mediana de 37 años. El principal factor de riesgo fue neutropenia secundaria a patología onco-hematológica. Según criterios EORTC/MSG hubo 12 infecciones probables y seis probadas (Tabla 1 ).

La principal localización de estas infecciones fue pulmonar (Tabla 1).

Nueve pacientes fueron diagnosticados con GM, ocho con cultivos y uno con los dos métodos (Tabla 1). El promedio de GM realizados fue de 1,42 (1-3). De los 10 casos en que el GM fue positivo, la mayoría de ellos (7/10) tuvieron índices entre 0,5 y 1.

En los casos en los que se logró identificar el agente (9/18), Rhizopus oryzae fue el más frecuente; otras especies aisladas se detallan en la Tabla 1. Estos se obtuvieron principalmente por cultivo de tejidos (Tabla 1). En cinco de éstos se realizó GM, resultando sólo uno positivo.

La mayor parte de los pacientes fueron tratados con voriconazol, seguido por anfotericina y posaconazol (usados conjuntamente) (Tabla 2). La letalidad global en EFI a los 30 días fue de 38,8\% (7/18), y al compararla

Tabla 1. Características generales de los pacientes con EFI. Región de Valparaíso, Chile: años 2012-2014 (n: 18)

\begin{tabular}{|c|c|}
\hline Edad (mediana) & 37 \\
\hline Sexo masculino $(n)$ & 11 \\
\hline \multicolumn{2}{|l|}{ Patologías de base en pacientes } \\
\hline Onco-hematológica & 12 \\
\hline Lupus eritematoso sistémico (LES) & 4 \\
\hline Enfermedad pulmonar crónica & 1 \\
\hline SIDA & 1 \\
\hline \multicolumn{2}{|c|}{ Categorías diagnósticas EORTC/MSG } \\
\hline Probable & 12 \\
\hline Probada & 6 \\
\hline \multicolumn{2}{|l|}{ Localización de la infección } \\
\hline Pulmonar & 12 \\
\hline Senos paranasales & 4 \\
\hline Sangre & 2 \\
\hline \multicolumn{2}{|c|}{ Método de laboratorio diagnóstico } \\
\hline GM & 9 \\
\hline $\mathrm{GM}+$ cultivo de tejidos & 1 \\
\hline Cultivo de tejidos + BP & 3 \\
\hline Cultivo de LBA + EMD & 3 \\
\hline Hemocultivo & 2 \\
\hline \multicolumn{2}{|l|}{ Especies aisladas } \\
\hline Rhizopus oryzae & 3 \\
\hline Lichtheimia corymbifera & 1 \\
\hline Sarocladium strictum & 1 \\
\hline Sarocladium kiliense & 1 \\
\hline Alternaria alternata & 1 \\
\hline Aspergillus fumigatus & 1 \\
\hline Micelio estéril & 1 \\
\hline
\end{tabular}


Tabla 2. Antifúngicos utilizados en el tratamiento de los pacientes con EFI

\begin{tabular}{|ll|}
\hline Antifúngico & $\mathbf{n}$ \\
\hline Voriconazol & 9 \\
Anfotericina B*/posaconazol & 3 \\
Anfotericina B y voriconazol & 2 \\
Caspofungina y voriconazol & 1 \\
Anfotericina liposomal & 1 \\
Caspofungina/posaconazol & 1 \\
\hline Anfotericina B & 1 \\
\hline *deoxicolato. & \\
\hline
\end{tabular}

con el período $2004-2009^{3}$, donde fue de $86,6 \%$ (13/15), hubo una reducción de $47,8 \%$, que fue estadísticamente significativa (p: 0,01).

\section{Discusión}

Los casos de EFI por hongos filamentosos diagnosticados en dos hospitales públicos de la Región de Valparaíso durante el período señalado ocurrieron en ambos sexos y en todos los grupos etarios. Esto coincide con lo publicado previamente en nuestra región ${ }^{3}$. La mayoría de los episodios $(66,6 \%)$ ocurrió en pacientes onco-hematológicos aunque se presentaron casos también en pacientes con otros factores de riesgo. En la serie publicada previamente en nuestra región, 50\% (7/14) de los pacientes eran oncohematológicos, cuatro tenían infección por VIH en etapa SIDA y cuatro, otros factores de riesgo (cetoacidosis diabética, fibrosis quística y uso de peritoneo-diálisis) ${ }^{3}$. Esto coincide con otras experiencias nacionales e internacionales, donde los pacientes con neutropenia secundaria al tratamiento de leucemia mieloide presentan el mayor riesgo para adquirir estas infecciones ${ }^{5,6,14}$. De los cuatro casos con mucormicosis, tres ocurrieron en pacientes con lupus eritematoso diseminado-LES, lo que es distinto a otras publicaciones, donde la cetoacidosis diabética es el principal factor de riesgo $0^{3,15,16}$.

El diagnóstico de EFI por hongos filamentosos fue realizado principalmente por detección de GM, por lo que muchos diagnósticos se debieron de catalogar de probables, según los criterios de EORTC/MSG ${ }^{11}$. Esto es distinto a lo publicado anteriormente en la región, donde todos los casos ( 9 probables y 5 probados) se diagnosticaron con cultivos, ya que no existía un protocolo estructurado de diagnóstico de laboratorio, con tiempos breves para informe y que además incluyera la detección de $\mathrm{GM}^{3}$.

El número promedio de pruebas de GM realizada a cada paciente fue de $1,29^{1-3}$. Esto es insuficiente, en varias guías se considera necesario realizar una curva de índices más que un solo valor aislado, debido a que favorece un mejor diagnóstico. La medición seriada de GM permite además vigilar la respuesta terapéutica al antifúngico indicado ${ }^{10,17}$.

Los agentes aislados con mayor frecuencia en los cultivos pertenecieron a los géneros Rhizopus y Sarocladium, los que no producen elevación del índice de GM como Aspergillus. En cambio, la casi totalidad de los casos de aspergilosis se diagnosticaron con GM, a diferencia del período anterior donde no se contaba con esta técnica ${ }^{3,5}$.

En el tratamiento de la aspergilosis, el antifúngico más utilizado fue voriconazol, seguido por anfotericina y posaconazol usados conjuntamente para el tratamiento de la mucormicosis. Esto es distinto al período 2004-2009, donde anfotericina $\mathrm{B}$ deoxicolato fue el más utilizado, tanto para aspergilosis como para la infección por otros hongos filamentosos ${ }^{3}$. Herbretch y cols., publicaron que en aspergilosis invasora, voriconazol se asocia a menor mortalidad que el uso de anfotericina deoxicolato ${ }^{18}$.

La terapia antifúngica preventiva guiada por TAC $^{19} \mathrm{O}$ por un marcador sérico como $\mathrm{GM}^{20}$ es de utilidad y puede ser un sustituto eficaz para la terapia empírica y de esta forma evitar la indicación innecesaria de antifúngicos.

La letalidad global a 30 días de realizado el diagnóstico fue de $38,8 \%$ (7/18), menor al 86,6\% (13/15) del período anterior en nuestra región ${ }^{3}$, siendo comparable con cifras de otras publicaciones ${ }^{6,21}$. Esta disminución se podría explicar, entre otros factores, por la sospecha precoz, la implementación del diagnóstico rápido estructurado y con plazos pre-establecidos para los informes, el uso de antifúngicos de mayor eficacia y por diferencias de co-morbilidad entre las dos series.

El presente trabajo puede presentar sesgos intrínsecos al diseño de estudio; sin embargo, muestra que en relación al período anterior en nuestra región, manteniéndose las características biodemográficas de los pacientes con EFI, pero con un cambio en la forma de pesquisarlas, la letalidad global asociada se redujo ostensiblemente.

\section{Resumen}

Introducción: la enfermedad fúngica invasora (EFI) por hongos filamentosos es una causa frecuente de morbilidad y mortalidad en pacientes inmunocomprometidos, en especial en aquellos con leucemia mieloide. En el 2011 se implementó en la Región de Valparaíso un protocolo de diagnóstico rápido de la EFI por hongos filamentosos. Objetivos: describir los casos de EFI por hongos filamentosos de la Región de Valparaíso, desde la implementación del diagnóstico rápido y compararlos con el período 2004-2009. Materiales y Método: Estudio descriptivo y 
prospectivo realizado en los hospitales públicos Carlos van Buren de Valparaíso y Gustavo Fricke de Viña del Mar. Se seleccionaron aquellos pacientes con diagnóstico de EFI por hongos filamentosos considerando los criterios EORTC/MSG. Se obtuvieron datos demográficos, enfermedad de base, factores de riesgo para EFI, resultados de galactomanano (GM), cultivos y biopsias, tratamiento y letalidad global a 30 días. Resultados: Se identificaron 18 pacientes, seis con EFI probadas y 12 probables. Nueve fueron diagnosticados con galactomanano, ocho con cultivos y uno con los dos métodos. En los casos en que se aisló el agente (9/18), Rhizopus oryzae fue el más frecuente. Al comparar la letalidad global con la del período 2004-2009, hubo una reducción de 47,8\%, la cual fue estadísticamente significativa. Conclusiones: En relación a lo publicado anteriormente en la región, se conservan las características demográficas y de co-morbilidad de los pacientes con EFI por hongos filamentosos; sin embargo, la introducción del nuevo protocolo de diagnóstico rápido se asoció a una disminución en la letalidad global.

\section{Referencias bibliográficas}

1.- Pemán J, Salavert M. Epidemiología general de la enfermedad fúngica invasora. Enferm Infecc Microbiol Clin 2012; 30 (2): 90-8.

2.- Siwek G T, Pfaller M A, Polgreen P M, Cobb S, Hoth P, Magalhaes-Silverman M, et al. Incidence of invasive aspergillosis among allogeneic hematopoietic stem cell transplant patients receiving voriconazole prophylaxis. Diagn Microb Infect Dis 2006; 55: 209-12.

3.- Cruz R, Piontelli E. Enfermedad fúngica invasora en pacientes de cinco hospitales de la Región de Valparaíso, Chile: 2004 a 2009. Rev Chilena Infectol 2011; 28: 123-9.

4.- Singh N, Paterson L. Aspergillus infections in transplant recipients. Clin Microbiol Rev 2005; 18: 44-69.

5.- Cruz R, Vieille P, Fuentes D, Ponce E, Piontelli E. Frecuencia de micosis pulmonares determinadas mediante la medición de secreciones pulmonares. Rev Med Chile 2012; 150 (5): 595-601.

6.- Rabagliati R, Fuentes G, Guzmán A M, Orellana E, Oporto J, Aedo I, et al. Enfermedad fúngica invasora en pacientes hematooncológicos y receptores de trasplante de precursores hematopoyéticos bajo la perspectiva de los criterios diagnósticos EORTC/MSG. Rev Chilena Infectol 2009; 26 (3): 212-9.

7.- Husain S, Alexander B D, Munoz P, Avery R K, Houston S, Pruett T, et al. Opportunistic mycelial fungal infections in organ transplant recipients: Emerging importance of non-Aspergillus mycelial fungi. Clin Infect Dis 2003; 37: 221-9.

8.- Roden M M, Zaoutis T E, Buchanan W L,
Knudsen T A, Sarkisova T A, Schaufele R L, et al. Epidemiology and outcome of Zygomycosis: a review of 929 reported cases. Clin Infect Dis 2005; 41 (5): 634-53.

9.- Caillot D, Casanovas O, Bernard A, Couailler J F, Durand C, Cuisenier B, et al. Improved management of invasive aspergillosis in neutropenic patients using early thoracic computed tomographic scan and surgery. J Clin Oncol 1997; 15: 139-47.

10.- Cruz R. Guía para el diagnóstico de laboratorio de enfermedad fúngica invasora por hongos filamentosos. Rev Chilena Infectol 2014; 31: 173-9.

11.- De Pauw B, Walsh T J, Donnelly J P, Stevens D A, Edwards J E, Calandra T, et al. Revised definitions of invasive fungal disease from the European Organization for Research and Treatment of Cancer/Invasive Fungal Infections Cooperative Group and the National Institute of Allergy and Infectious Diseases Mycoses Study Group (EORTC/MSG) Consensus Group. Clin Infect Dis 2008; 46 (12): 1813-21.

12.- Maertens J, Maertens V, Theunissen K, Meersseman W, Meersseman P, Meers S, et al. Bronchoalveolar lavage fluid galactomannan for the diagnosis of invasive pulmonary aspergillosis in patients with hematologic diseases. Clin Infect Dis 2009; 49 (11): 1688-93.

13.- Pemán J. ¿Qué se puede esperar del diagnóstico micológico clásico de la aspergilosis invasora? Aspergilosis Invasora. Guía de bolsillo. Editor: José Pontón 2003; 47-50.

14.- Pagano L, Caira M, Candoni A, Offidani M, Fianchi L, Martino B, et al .The epidemiology of fungal infections in patients with hematologic malignancies: the SEIFEM-2004 study. Haematologica 2006; 91: 1068-75.

15.- Romero-Zamora J L, Bonifaz A, Sánchez C J, Lagunas-Ramírez A, Hidalgo-Loperena $\mathrm{H}$. Mucormicosis rinocerebral. Reporte de doce casos. Rev Med Hosp Gen (Mex) 2000; 63: 178-84.

16.- Bravo M, Ferrer S, Etchart M, Trujillo S. Mucormicosis rinocerebral. Comunicación de cuatro casos. Rev Med Chile 1999; 127: 712-8.

17.- Pinel C, Fricker-Hidalgo H, Lebeau B, Garban F, Hamidfar R, Ambroise-Thomas P, et al. Detection of circulating Aspergillus fumigates galactomannan: value and limits of the Platelia test for diagnosing invasive aspergillosis. J Clin Microbiol 2003; 41: 2184-6.

18.- Herbrecht R, Denning D W, Patterson T F, Bennett J E, Greene R E, OestmannJörg-W, et al. Voriconazole versus amphotericin $\mathrm{B}$ for primary therapy of invasive aspergillosis. $\mathrm{N}$ Engl J Med 2002; 347: 408-15.

19.- Maertens J, Theunissen K, Verhoef G, et al. Galactomannan and computed tomographybased preemptive antifungal therapy in neutropenic patients at high risk for invasive fungal infection: a prospective feasibility study. Clin Infect Dis 2005; 41 (9): 1242-50.

20.- Leventakos K, Lewis R, Kontoyiannis D. Fungal infections in leukemia patients: how do we prevent and treat them? Clin Infect Dis 2010; 50 (3): 405-15.

21.- Upton A, Kirby K, Carpenter P, Bocckh M, Marr K. Invasive aspergillosis following hematopoietic cell transplantation: outcomes and prognostic factors associated with mortality. Clin Infect Dis 2007; 44: 531-40. 